\title{
REFLUJO VESICOURETERAL TRAS TRASPLANTE RENAL EN LA EDAD PEDIÁTRICA
}

Rafael Barrero Candau, Miguel Fernández Hurtado, Francisco García Merino, Julia Fijo López-Viota' ', Eduardo León Dueñas² y Francisco Torrubia Romero².

Servicio de Cirugía Pediátrica. Servicio de Nefrología Pediátrica'. Servicio de Urología². Hospitales Universitarios Virgen del Rocío. Sevilla. España.

Resumen.- OBJETIVO: Analizamos la frecuencia de reflujo vesicoureteral tras trasplante renal (TR) en edad pediátrica y los factores que lo favorecen.

MÉTODOS: Entre 1978 y 2006 se realizaron en nuestro centro 201 TR pediátricos. En un análisis retrospectivo se revisó la prevalencia de RVU postrasplante. En aquellos que precisaron cirugía correctora de RVU postrasplante se analizó: diuresis residual pretrasplante, patología previa al TR o adquirida tras éste relacionada con RVU. Además se analizó forma de presentación, si el reflujo era al injerto o a los riñones nativos, grado de éste y técnica quirúrgica empleada para corregirlo.

RESULTADOS: Doce pacientes (5,9\%) precisaron cirugía antireflujo. En 10 el reflujo fue al injerto y en 2 al riñón nativo. El reflujo se presentó como infección del tracto urinario (ITU) en 11 casos y deterioro progresivo de la función del injerto en 1. En 10 pacientes se detectó patología uretrovesical fa- vorecedora de reflujo vesicoureteral (vejigas no compliantes, hiperactividad del detrusor (HDD), válvulas de uretra posterior (VUP), estenosis de uretra, etc..). Tras ampliación del túnel submucoso desaparecieron las ITU en los 12 casos, mientras que la C.U.M.S a los 6 meses mostró resolución en 10 casos y disminución del grado de reflujo en 2.

CONCLUSIÓN: El mayor porcentaje de reflujo vesico-ureteral postrasplante en la edad pediátrica, se relaciona con la mayor frecuencia de patología uretrovesical en los niños receptores del trasplante. La realización de un túnel submucoso más amplio durante el implante, en aquellos niños con patología uretrovesical pretrasplante, podría reducir el porcentaje de esta complicación.

Palabras clave: Niños. Reflujo vesicoureteral. Trasplante renal.

Summary.- OBJECTIVES: We analyze the frequency of vesicoureteral reflux and the factors that favor its appearance after kidney transplantation in pediatric patients.

METHODS: This retrospective analysis examined the prevalence of posttransplant vesicoureteral reflux in a total of 201 kidney transplants performed in children at our centre between 1978 and 2006. In patients who required corrective surgery for this problem, we analyzed pretransplant residual diuresis, pretransplant pathology and posttransplant problems related to vesicoureteral reflux. We also analyzed the form of presentation, whether reflux was to the graft or to the native kidney, degree of reflux, and surgical technique used to correct reflux.

RESULTS: Twelve patients (5.9\%) needed surgery to correct reflux to the graft (10 children) or to the native kidney 12 children). Reflux presented as urinary tract infection in 11 children and progressive graft failure in 1. Urethrovesical disorders that favoured vesicoureteral reflux were present in 10 patients 
Inoncompliant bladder, detrusor overactivity, posterior urethral valves, urethral stenosis). Lengthening the submucosal tunnel stopped urinary tract infections in all 12 patients, whereas 6-month voiding cystourethrograms showed resolution in 10 patients and reduction in the degree of reflux in 2.

CONCLUSION: The high percentage of posttransplant vesicoureteral reflux in pediatric patients was related with higher frequency of vesicourethral pathology in children who received the transplant. In children with pretransplant urethrovesical anomalies we recommend an initial technique which utilizes a longer submucosal tunnel during implantation, such as the Lich-Gregoir.

Keywords: Child. Renal Transplantation. Vesico-Ureteral reflux.

\section{INTRODUCCIÓN}

El trasplante renal es el tratamiento de elección en la insuficiencia renal crónica terminal. En la edad pediátri$c a$, además de evitar las complicaciones de la diálisis y dar una mayor calidad de vida, permite un mejor crecimiento y desarrollo (1).

El reflujo vesicoureteral (RVU) primario en la edad pediátrica es una enfermedad prevalente que produce infecciones urinarias de repetición, pielonefritis, nefropatía tubulointersticial, HTA e insuficiencia renal (2).

El RVU tras el trasplante renal puede ser a los riñones nativos y/o al propio injerto renal. Sus consecuencias son las mismas que las del reflujo vesicoureteral primario, aunque las pielonefritis sobre los injertos renales (habitualmente procedentes de cadáveres adultos) producen nefropatía por reflujo con menos frecuencia que las pielonefritis sobre riñones infantiles en desarrollo (3).

La frecuencia de RVU postrasplante en la edad pediátrica, y cuales son los factores que favorecen el desarrollo de RVU, son aspectos poco conocidos y en los que actualmente existe controversia entre los trabajos publicados (3-8).

Analizamos la frecuencia de RVU postrasplante en la edad pediátrica, así como los factores que lo favorecen, en aquellos niños que precisaron actuación quirúrgica para corregirlo.

\section{MATERIAL Y MÉTODO}

Entre 1978 y 2006 se realizaron en nuestro centro 201 TR en edad pediátrica. En un análisis retrospectivo se revisó la prevalencia de RVU postrasplante. En los niños con RVU postrasplante se analizaron los factores que se han sugerido que están en relación con RVU (3-8): diuresis residual pretrasplante, $\mathrm{HDD}$, disminución de la complianza vesical, VUP, diabetes, obstrucción al flujo urinario de salida (estenosis de uretra, disinergia detrusor esfinteriana,...). También se revisó la forma de presentación del RVU, lugar al que refluyó (al injerto o a los riñones nativos), grado del reflujo y técnica quirúrgica empleada para corregirlo.

Para la graduación del reflujo a los riñones nativos se empleó la clasificación internacional de enfermedades (CIE-10), mientras que cuando afectaba al injerto renal se dividió simplemente en RVU con dilatación ureteral o sin ésta, ya que la hipotonía ureteral e implantación ureteral ectópica del riñón trasplantado interfieren en la correcta gradación del RVU.

Tras revisar los estudios urodinámicos previos a la corrección del RVU postrasplante se asignó un diagnóstico urodinámico según los términos y valores definidos por la Guía de la Sociedad Internacional de Continencia $(9,10)$.

El protocolo seguido en nuestro centro para el reimplante ureteral del trasplante es el siguiente: La técnica utilizada durante el trasplante es la descrita por CamposFreire y cols. (11). Sólo se tutoriza la anastomosis ureterovesical en aquellos casos en los que se considera riesgo elevado de fístula, utilizando entonces catéter ureteral externo, que se retira, el séptimo día postoperatorio, aunque en los últimos años se esta dejando tutorizado en casi todos. Mientras que el protocolo utilizado en el seguimiento es el siguiente: no se realiza cistouretrografía miccional seriada (CUMS) postrasplante de forma sistemática, sólo en aquellos casos con ITU de repetición o pielonefritis aguda. En los pacientes con ITU de repetición en los primeros meses postrasplante se instaura profilaxis antibiótica durante 6 meses. Si aparece ITU a pesar de la profilaxis antibiótica, tras suspender ésta o acompañada de dilatación de la vía se realiza CUMS. El estudio urodinámico sólo se realiza si se confirma RVU en la CUMS (Cuadro 1). En RVU de bajo grado a riñones nativos se adopta una actitud poco agresiva, realizando profilaxis antibiótica, tratamiento anticolinérgico (si existe baja acomodación) e inyecciones submeatales endoscópicas. Cuando persiste el RVU o reaparecen pielonefritis a pesar del tratamiento se realiza nefroureterectomía. En RVU de alto grado o con uréteres extravesicalizados, de riñones no funcionantes se realiza directamente nefroureterectomía.

En el RVU de bajo grado, al injerto inicialmente profilaxis antibiótica y en aquellos casos con HDD o disminución de la complianza vesical se añadió anticolinérgicos orales. Cuando se producen ITU de repetición o pielonefritis agudas (PNA), a pesar de la profilaxis y el tratamiento anticolinérgico realizamos cirugía abierta reconstructiva.

La sistemática seguida para la corrección mediante cirugía abierta es la siguiente: se realiza uretrocistoscopia en la que a la vez que se descartan restos valvulares y otras patologías uretrovesicales que pudieran estar condicionando altas presiones vesicales, se coloca catéter ureteral en el uréter trasplantado (no siempre posible) y en el uréter nativo ipsilateral al injerto. Incisión sobre la cicatriz del trasplante, identificación del catéter e intento de ampliación del túnel submucoso del uréter según técnica de Linch-Gregoire. Si se producen accidentes quirúrgicos por 
CUADRO 1. ESQUEMA QUE SEGUIMOS EN EL MANEJO DEL REFLUJO VESICOURETERAL POSTRASPLANTE EN EL NIÑO.

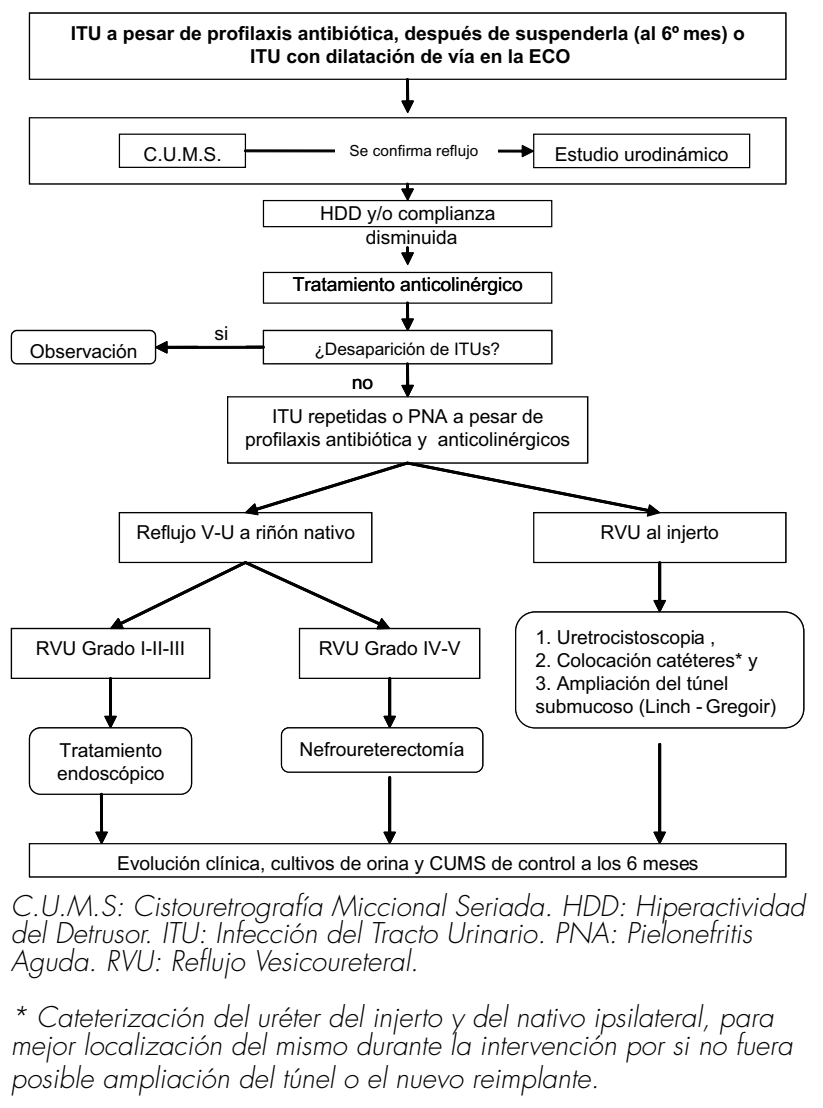

la intensa fibrosis o imposibilidad de ampliación del túnel se intenta primero nuevo reimplante tipo Campos-Freire y si la longitud y diámetro del uréter no lo permiten ureteroureterostomía o pieloureterostomía con el sistema excretor nativo ipsilateral.

\section{RESULTADOS}

\section{Frecuencia de reflujo vesicoureteral}

Doce pacientes ( 9 niños y 3 niñas) presentaron RVU postrasplante. La edad media a la que se realizó el trasplante fue 11.2 (rango: 3 - 16) años y la edad media a la que se realizó el diagnóstico de reflujo vesicoureteral postrasplante fue 12.1 (rango: 4 - 18) años, siendo el tiempo medio desde el trasplante renal al diagnóstico de reflujo de 8 (rango: 2 - 23) meses. En 10 casos el reflujo fue al injerto y sólo en 2 a los riñones nativos.

\section{Factores favorecedores de RVU postrasplante}

Entre las patologías previas al trasplante que podrían haber contribuido al desarrollo posterior de reflujo vesicoureteral se observó (Tabla I): antecedentes de válvulas uretrales posteriores en 7 casos; reflujo vesicoureteral en 5 casos, en dos de ellos se había realizado cirugía de reimplante transvesical, según técnica de Cohen, mientras que en los otros tres habían desaparecido en la CUMS realizada en el estudio pretrasplante; estenosis de uretra en 2 casos, una tras reparación de hipospadias y otra tras

TABLA I. PATOLOGÍA PRETRASPLANTE, DIURESIS RESIDUAL PRETRASPLANTE Y HALLAZGOS URODINÁMICOS EN NIÑOS CON RVU POSTRASPLANTE.

\begin{tabular}{|c|c|c|c|c|}
\hline $\mathrm{n}$ & Sexo & $\begin{array}{l}\text { Patología pretrasplante que } \\
\text { pudiera condicionar RVU posterior }\end{array}$ & $\begin{array}{c}\text { Diuresis residual } \\
\text { pretrasplante }(\mathrm{mL} / 24 \mathrm{~h})\end{array}$ & $\begin{array}{l}\text { Estudio urodinámico } \\
\text { tras diagnóstico de RVU }\end{array}$ \\
\hline 1 * & V & RVU y Reimplante ureteral bilateral. & 1.560 & postrasplante \\
\hline 2 & V & VUP & 2.000 & Vejiga compliante \\
\hline 3 & M & No se encontró. & 150 & HDD \\
\hline 4 & V & $\begin{array}{l}\text { RVU, VUP y Reimplante ureteral bilateral } \\
\text { Retrasplante (tras el } 4^{\circ} \text { trasplante) }\end{array}$ & $\begin{array}{c}500 \\
0\end{array}$ & $\begin{array}{l}\mathrm{HDD} \\
\mathrm{HDD}\end{array}$ \\
\hline 5 & $M$ & No se encontró & 1.850 & Vejiga compliante \\
\hline 6 & $\mathrm{~V}$ & RVU y VUP & - & $\mathrm{HDD}$ \\
\hline 7 & M & RVU & 560 & Vejiga compliante \\
\hline 8 & V & $\begin{array}{l}\text { RVU y VUP. Retrasplante (tras el } 3^{\text {er }} \text { trasplante) } \\
\text { Estenosis uretral }\end{array}$ & 750 & HDD \\
\hline 9 & V & VUP & 2.200 & - \\
\hline 10 & $\mathrm{~V}$ & Hipospadias. Estenosis de uretra. & 1.800 & Vejiga compliante \\
\hline 11 & V & VUP & - & Vejiga compliante \\
\hline 12 & V & VUP & - & \\
\hline
\end{tabular}

HDD: Hiperactividad del detrusor. M: Mujer. RVU: Reflujo vesicoureteral. V: Varón. VUP: Válvulas de uretra posterior. Vejiga compliante: Presión a la capacidad vesical teórica < de $15 \mathrm{~cm}$. H2O. * En este caso el reflujo vesico-ureteral fue al riñón nativo derecho.

- Diuresis residual no registrada o estudio urodinámico no realizado. 
resecciones repetidas de válvulas uretrales posteriores. En 2 casos el RVU no se produjo tras un primer trasplante, uno fue tras un tercer trasplante y otro tras un cuarto trasplante. Tan solo en dos casos no se encontró antecedente alguno que justificara el reflujo. La diuresis residual media pretrasplante fue de $1.052 \mathrm{ml}$ [0 - 2.200] encontrando reflujo independiente del volumen de diuresis residual. En los estudios urodinámicos se observó hiperactividad del detrusor y vejiga con mala acomodación en 5 casos, de los que cuatro eran pacientes con antecedentes de VUP.

\section{Forma de presentación - Grado de reflujo - PNA - Disfun- ción del injerto}

La forma de presentación en 11 casos fue ITU de repetición, con episodios repetidos de pielonefritis aguda y en un caso deterioro de la función renal asociado a dilatación de la vía. Todos los injertos eran procedentes de cadáver. Tras los episodios de ITU se produjo ascenso transitorio de la cifra de creatinina en 6 de los 12 pacientes que presentaron RVU postrasplante, y en 2 casos se mantuvo el ascenso de la cifra de creatinina plasmática. Ningún injerto se perdió por éste motivo.

La CUMS confirmó el diagnóstico en todos los casos y mostró que el reflujo era al injerto en 10 casos y a los riñones nativos en otros dos. Nueve de los reflujos al injerto eran sin dilatación mientras que el otro presentaba dilatación de la vía. El reflujo a los riñones nativos fue bilateral grado $V$ en uno y bilateral grado II en el otro.

\section{Manejo del RVU postrasplante}

Los pacientes con diagnóstico de hiperactividad del detrusor o baja complianza fueron tratados con oxibutinina a $0,2 \mathrm{mg} / \mathrm{Kg}$. No se consiguió la desaparición del reflujo ni las ITU en ningún caso.

Como tratamiento quirúrgico del RVU se realizó ampliación extravesical del túnel submucoso según técnica de Linch-Gregoire en 7 casos, nuevo reimplante tipo Campos-Freire en 2 y anastomosis de la pelvis del injerto al uréter nativo en otro. El reflujo grado $\mathrm{V}$ al riñón nativo se trató mediante nefroureterectomía, mientras que el de grado II se realizó mediante inyección submeatal endoscópica de Deflux®.

El control postintervención mostró desaparición de los episodios de ITU en los 12 casos, mientras que la C.U.M.S a los 6 meses evidenció corrección total del reflujo vesicoureteral en 10 casos y mejora del grado de reflujo en 2, que no han presentado nuevas ITU a pesar de la retirada de la profilaxis antibiótica. En uno de los casos en los que no se corrigió completamente el RVU lo único que se encontró como posible factor favorecedor del reflujo fue una flujometría pobre (parcialmente obstructiva) tras reparación de la estenosis de uretra posthipospadias. Mientras que en el otro caso no se evidenció factor alguno que favoreciera la persistencia del reflujo.

\section{DISCUSIÓN}

\section{Frecuencia de $R V U$ postrasplante}

Al igual que otros grupos no descartamos de for- ma sistemática el RVU postrasplante. Realizamos estudio sólo cuando aparece ITU a pesar de la profilaxis antibiótica, tras retirar ésta a los 6 meses o ITU con dilatación de la vía en la ecografía; de forma que habitualmente el que se diagnostica suele precisar tratamiento quirúrgico. La prevalencia de RVU postrasplante intervenidos en los adultos de nuestra serie (14) y en las de otros grupos $(12,13)$ es $<1 \%$, mientras que la prevalencia observada en la edad pediátrica ha sido 5,5\%. La mayor prevalencia de RVU postrasplante en niños también ha sido observada por otros autores que realizan trasplante en adultos y niños (36) que refieren un $34-36 \%$ de RVU postrasplante. La menor frecuencia de nuestra serie con respecto a otros grupos se explica por que el estudio para descartar RVU sólo se realizó en los casos en los que se produjo ITU de repetición y no de forma sistemática.

Respecto a la baja prevalencia observada de RVU postrasplante a riñones nativos (2 casos de 201) creemos que puede deberse a la corrección sistemática de todo RVU detectado en el estudio pretrasplante además del estudio no sistemático ya mencionado. Pensamos que esta actitud evita posibles pielonefritis posteriores que estarían agravadas por la inmunosupresión que realizan los pacientes trasplantados. El niño que precisó nefroureterectomía por RVU grado $V$ al riñón nativo ya presentaba RVU en el estudio pretrasplante, pero al ser monorreno y con una buena diuresis residual se decidió mantenerlo para no desfuncionalizar la vejiga antes del trasplante. En el otro caso al ser bilateral y de grado II se decidió inyección submeatal endocópica.

\section{Factores que favorecen reflujo vesicoureteral postrasplante}

La presencia de RVU puede depender del momento tras el trasplante en el que se realiza el estudio y del tipo de anastomosis ureterovesical realizada $(4,12,15)$. En un estudio prospectivo randomizado (16), el RVU fue menos frecuente cuando se realizó la técnica de Leadbetter-Politano. No obstante y al igual que la mayoría de los grupos de trasplante realizamos abordaje extravesical por su menor índice de complicaciones postoperatorias, necesidad de un segmento ureteral más corto y menor tiempo quirúrgico para realizarlo.

Por otro lado la alta frecuencia de patología uretrovesical observada, en 10 de los 12 pacientes, podría haber sido la causa o el factor favorecedor del mayor porcentaje de RVU (Tabla I). Esta observación es congruente con el hecho de que la patología que produce la insuficiencia renal crónica (I.R.C.) en el niño es distinta a la que la produce en adultos. Sin embargo se estima que menos del $5 \%$ de los pacientes con I.R.C. presentan como patología de base una alteración estructural de la vía urinaria (17).

Se ha sugerido que aquellos pacientes con baja diuresis residual y que presentan una vejiga desfuncionalizada, tienen mayor frecuencia de reflujo vesicoureteral. Ya que una vejiga desfuncionalizada dificulta la realización de un túnel submucoso suficiente durante el trasplante y condiciona presiones altas tras la refuncionalización. Nosotros al igual que otros grupos (3) no observamos correlación entre el volumen de diuresis residual pretrasplante y la aparición de RVU postrasplante. 


\section{RVU - ITU - PNA - Disfunción del injerto}

Las ITU son frecuentes después de un TR, aproximadamente el $60 \%$ de los pacientes sufren al menos un episodio de ITU $(15,18)$ habitualmente en los primeros meses tras el trasplante. Según diversos autores $(12,13,15,18$ 22) la presencia de RVU no aumenta la frecuencia de ITU después del trasplante, sin embargo si se han esbozados diversos factores de riesgo como son diabetes $(12,23) \mathrm{ca}$ teterismo vesical y la I.R.C.T secundaria VUP (12). Las VUP también destacan en nuestro estudio como posible factor de riesgo de RVU postrasplante. Los pacientes con válvulas uretrales posteriores a pesar de mantener una capacidad vesical media aceptable presentaron una baja acomodación vesical, es decir presentaron presiones vesicales elevadas durante la fase de llenado vesical.

Las pielonefritis postrasplante son raras en adultos pero son bastante frecuentes en niños (3). El RVU se ha asociado con aumento del riesgo de PNA (5), no obstante Thomalla y cols. (24) no encontraron RVU en 18 trasplantados adultos con PNA que se confirmó en otros estudios.

Por otro lado los injertos renales procedente de adultos sufren raramente nefropatía por reflujo (6) y según French y cols. (25) el reflujo vesicoureteral no afecta la función del injerto a medio y largo plazo. En los 12 casos los injertos eran procedentes de cadáveres adultos, no se perdió ninguno de ellos por este motivo (las ITU o pielonefritis de repetición), aunque si produjo ascenso transitorio de creatinina en 6 casos, siendo este ascenso mantenido en 2 .

\section{Manejo y técnica quirúrgica a realizar}

El alto porcentaje de resolución del RVU observado tras la ampliación del túnel submucoso, y la alta incidencia de patología uretrovesical (10/12) en los pacientes con RVU postrasplante sugiere realizar de entrada un túnel submucoso más amplio en aquellos pacientes con antecedentes de patología uretrovesical (26). Un reimplante más lateral y menos posterior, también facilita la cateterización endoscópica del uréter y en casos muy seleccionados las inyecciones submeatales endoscópicas, aunque la efectividad demostrada por éstas hasta la fecha ha sido baja (27).

En resumen el mayor porcentaje de reflujo vesicoureteral postrasplante en la edad pediátrica, se relaciona con la mayor frecuencia de patología uretrovesical en los niños receptores del trasplante. La realización de un túnel submucoso más amplio durante el implante, en aquellos niños con patología uretrovesical pretrasplante, podría reducir el porcentaje de esta complicación.

\section{AGRADECIMIENTOS}

A D. Pedro Montañés Medina motor del trasplante renal en nuestro centro y maestro de todos los miembros del actual equipo de trasplante, por su labor a lo largo de todos estos años.

A los miembros del Servicio de Nefrología Pediátrica, por su dedicación a la continua mejora del programa de trasplante renal infantil.

\section{BIBLIOGRAFÍA Y LECTURAS RECOMENDADAS (*lectura de interés $y^{* *}$ lectura fundamental)}

1. BARNETT, C.C.; PARTRICK, D.A.; MAY, D.J.: "Update in pediatric trasplantation". Curr. Opin. Urol., 7: 103, 1997.

2. HODSON, E.M.; WHEELER, D.M.; VIMALACHANDRA, D. y cols.: "Intervenciones para el reflujo vesicoureteral primario". La Cochrane Library. Oxford: Update Software. $\mathrm{N}^{\circ}$ 4. 2007.

3. RANCHIN, B.; CHAPUIS, F.; DAWHARA, M. y cols.: "Vesicouretral reflux after kidney transplantation in children". Nephrol. Dial. Transplant., 15: 1852, 2000.

4. HANEVOLD, C.D.; KAISER, B.A.; PALMER, J.A. y cols.: "Vesicoureteral reflux and urinary tract infections in renal transplant recipients". Am. J. Dis. Child., 141: 982, 1987.

5. DUNN, S.P.; VINOCUR, C.D.; HANEVOLD, C. y cols.: "Pyelonephitis following pediatric renal transplant: Increased incidente with vesicoureteral reflux". J. Pediatr. Surg., 22: 1095, 1987.

6. FONTANA, I.; GINEVRI, F.; ARCURI, V. y cols.: "Vesicoureteral reflux in pediatric kidney transplants: Clinical relevante to graft and patient outcome". Pediatr. Trasplant., 3: 206, 1999.

7. NEUHAUS, T.J.; SCHWÖBEL, M.; SCHLUMPF, R. y cols.: "Pyelonephritis and vesicoureteral reflux after renal transplantation in young children". J. Urol., 1400, 1997.

8. ZAONTZ, M.R.; HATCH, D.A.; FIRLIT, C.F.: "Urological complications in pediatric renal transplantation: Management and prevention”. J. Urol., 140: 1123, 1988.

9. ABRAMS, P.; CARDOZO, L.; FALL, M. y cols.: "The standardisation of terminology of lower urinary tract function: Report from the standardisation subcommitte of the international continence society". Neurourol. Urodyn., 21: 167, 2002.

10. EL GRUPO ESPAÑOL DE URODINÁMICA Y DE LA SINUG.: "Consenso sobre terminología y conceptos de la función del tracto urinario inferior". Actas Urol. Esp., 29: 16, 2005.

11. WOODRUFF, M.W.; BACHRACH, P.; CORICA, A. y cols.: "Ureteroneocystostomy in renal transplant". Urology, 1: 414, 1974.

12. CUVELIER, R.; PIRSON, Y.; ALEXANDER, G.P.J. y cols.: "Late urinary tract infection after transplantation: Prevalence, predisposition and morbidity". Nephron., 40: 76, 1985.

13. MASTROSIMONE, S.; PIGNATA, G.; MARESCA, M.C. y cols.: "Clinical significance of vesicoureteral reflux after kidney transplantation". Clin. Nephrol., 40: 38, 1993.

14. MONTAÑES MEDINA, P.; TORRUBIA ROMERO, F.J.; MARMOL NAVARRO, S. y cols.: "Lait Surgical complications in kidney transplantation. Experience and results". Arch. Esp. Urol., 42: 549, 1989.

15. MATHEW, T.H.; KINCAID-SMITH, P.; VIKRAMAN, P.: "Risks of vesicoureteric reflux in the transplanted kidney". N. Engl. J. Med., 297: 414, 1977.

16. WALTKE, E.A.; ADAMS, M.B.; KAUFFMAN, H.M. y cols.: "Prospective randomized comparison of urologic complications in end-to-side versus Politano-Lea- 
dbetter ureteroneocystostomy in 131 human cadáver renal transplants". J. Urol., 128: 1170, 1982.

17. US RENAL DATA SYSTEM.: "USRDS 1998 anual data report". The Nathional Institute of Health. Bethseda, MD. 1998.

18. PART, V.; HORCICHOVA, M.; MATOUSOVIC, K. y cols.: "Urinary tract infection in renal transplant patients". Infection, 13: 207, 1985.

19. BOOSTMA, M.; KOOTTE, A.M.M.; VAN BOCKEL, J.H. y cols.: "Clinical significance of vesicoureteral reflux into transplanted kindneys". Clin. Trasplant., 1: $311,1987$.

20. GRUNBERGER, T.; GNANT, M.; SAUTNER, T. y cols.: "Impact of vesico-ureteral reflux on graft survival in renal transplantation". Trasplant. Proc., 25: $1058,-1993$.

21. VIANELLO, A.; PIGNATA, G.; CALDATO, C. y cols.: "Vesico-ureteral reflux after kidney trasplantation: Clinical significance in the médium to long term". Clin. Nephrol., 47: 356, 1997.

22. MOLINA, G.; FEITOSA, L.C.; MARTIN, X. y cols.: "Incidente of vesicoureteral reflux after allograft renal transplantation". Transplant. Proc., 26: 292, 1994.

23. FOX, B.C.; SOLLIGER, H.W.; BELZER, F.O. y cols.: "A prospective randomized, double-blind study of trimethoprim-sulfamethoxazole for prophylaxis of infection in renal trasplantation: Clinical eficacy, absorption of trimethoprim-sulfamethoxazole, effect on the microflora, and the cost-benefit of prophylaxis". Am. J. Med., 89: 255, 1990.

24. THOMALLA, J.V.; GLEASON, P.; LEAPMAN, S.B. y cols.: "Renal transplant pyelonephritis". Clinical Trasplant., 2: 299, 1988.

25. FRENCH, C.G.; ACOTT, P.D.; CROCKER, J.F. y cols.: y cols.: "Extravesical ureteroneocystostomy with and without internalized ureteric stents in pediatric renal transplantation”. Pediatr. Transplant., 5: 21, 2001.

26. BARRERO, R.; FIJO, J.; FERNANDEZ-HURTADO, M.A. y cols.: "Vesicoureteral reflux after kidney transplantation in children". Pediatric. Tranplant. 11:498, 2007.

27. LATCHAMSETTY, K.C.; MITAL, D.; JENSIK, S. y cols.: "Use of collagen injections for vesicoureteral reflux in transplanted kidneys". Transplant. Proc., 35: 1378, 2003. 